\title{
The mechanism of blood concentrations of the Shenqi pill repairing injured epithelial cells of renal tubular in vitro
}

\author{
FEI HUANG ${ }^{1}$ and WENFENG MA ${ }^{2}$ \\ Departments of ${ }^{1}$ Nephrology and ${ }^{2}$ Pharmacy, The Central Hospital of Enshi Tujia \\ and Miao Autonomous Prefecture, Enshi, Hubei 445000, P.R. China
}

Received May 20, 2016; Accepted January 30, 2017

DOI: $10.3892 /$ etm.2017.4450

\begin{abstract}
In the present study, we investigated the best blood concentration of the mechanism of Shenqi pill repairing the injured epithelial cells of renal tubular in vitro. First, the injured hypoxia/reoxygenation model of rat proximal renal tubular epithelial cell strain (NRK-52E) was established. The animals were divided randomly into control, model, low concentration $(5 \mu \mathrm{g} / \mathrm{ml})$, moderate concentration $(10 \mu \mathrm{g} / \mathrm{ml})$ and high concentration $(20 \mu \mathrm{g} / \mathrm{ml})$ groups. The apoptotic rate was measured with flow cytometry and Jag2/Notch2/hes1 mRNA, and the protein expression was measured for 1, 3 and 7 days. It was found that in comparison to the control group, the growth of each group was prolonged with time, the levels of apoptosis, and the Jag2/Notch2/hes1 mRNA and protein expression decreased. Furthermore, the levels of the apoptotic rate, Jag2/Notch2/hes1 mRNA and protein expression of the moderate concentration and high concentration groups were significantly lower than those of the model and low-dose groups at each time-point $(\mathrm{P}<0.05)$. In conclusion, the Shenqi Pill alleviates the damage of renal tubular epithelial cells by inhibiting the Jag2/Notch2/hes1 signaling pathway; suitable concentration such as $10-20 \mu \mathrm{g} / \mathrm{ml}$ can exert protective effect.
\end{abstract}

\section{Introduction}

There is a higher incidence of acute kidney injury, for several reasons including ischemia and hypoxia, poisoning, infection, reperfusion, which may contribute to apoptosis or necrosis of renal tubular epithelial cells by the pathogenesis of inflammation, oxidative stress, calcium overload and metabolic

Correspondence to: Dr Wenfeng Ma, Department of Pharmacy, The Central Hospital of Enshi Tujia and Miao Autonomous Prefecture, 158 Wuyang Avenue, Enshi, Hubei 445000, P.R. China E-mail: mawenfeng2016@163.com

Key words: Shenqi pill, renal tubular epithelial cells, injured hypoxia/reoxygenation model, Jag2/Notch2/hes1 disorders of energy $(1,2)$. Notch 2 can be widely expressed in the development process of kidney, and then turn silent when mature, and can be activated to participate in the disease process in a variety of external stimulation rapidly and largely such as sugar, in ischemia reperfusion injury (3). The Notch inhibitor DAPT ( $\gamma$-inhibitor) has a protective effect on acute kidney injury (4). Jinguishenqi pill as an ancient prescription for tonifying kidney and kidney 'qi', $(5,6)$ has better application effect in acute and chronic renal injury disease.

We analyzed the repairing mechanism of Shenqi pill in vitro on acute injury of renal tubular epithelial cells, to find the optimal blood concentration.

\section{Materials and methods}

Main materials and reagents. Rat proximal renal tubular epithelial cell strains (NRK-52E) were purchased from Cell Resource of Shanghai Institutes for Biological Sciences Center of Chinese Academy of Sciences Committee on Type Culture Collection Cell Bank (Shanghai, China). Dulbecco's modified Eagle's medium (DMEM)/F12 medium powder, trypsin (Gibco, Grand Island, NY, USA), high pure nitrogen of high-pressure (Shanxi Taiyuan Pharmaceutical Co., Ltd., Taiyuan, China), total RNA extraction kit, polymerase chain reaction (PCR) primers, TranScript cDNA first-strand synthesis kit, SYBR-Green I real-time PCR kits, pre-stained protein molecular weight marker (Beijing Zhongshan Science and Technology Co., Ltd., Beijing, China), ECL chemiluminescence kit (Pierce Biotechnology, Inc., Rockford, IL, USA), BCA protein assay kit (Kaiji Biological Technology Development Co., Ltd., Nanjing, China), rabbit anti-human Jag2/Notch2/hes1 and $\beta$-actin monoclonal antibody (Santa Cruz Biotechnology, Inc., Santa Cruz, CA, USA), horseradish peroxidase-labeled goat anti-rabbit IgG (Sigma-Aldrich, St. Louis, MO, USA) were purchased.

Main instruments. HEPA-CLASS 100 three gas tissue incubator (Thermo Fisher Scientific, Waltham, MA, USA), inverted microscope (Leica, Mannheim, Germany), ESP Elite flow cytometer (Beckman Coulter, Fullerton, CA, USA), micropipette (Eppendorf AG, Hamburg, Germany), SW-CJ-2FD type double-sided clean bench (Zhejiang Sujing 
Purification Equipment Co., Ltd., Sujing, China), TGL-16B high-speed desktop centrifuge (Shanghai Anting Scientific Instrument Factory, Shanghai, China), cell culture flasks, culture plates (Corning Inc., Acton, MA, USA), 721 spectrophotometer (Third Shanghai Analytical Instrument Factory, Shanghai, China), DYCZ-40D mini transfer electrophoresis and WD-9465B horizontal shaker (Beijing Liuyi Instrument Factory, Beijing, China).

Animal model of ischemia/reperfusion. Conventional recovery, culture, subculture, frozen storage, cells which had been passaged 2-3 times and reached to $80 \%$ cell confluence after replaced with serum-free DMEM/F12 medium for $24 \mathrm{~h}$. The pure $\mathrm{N}_{2}$ was aerated continuously into oxygen-free liquid for $30 \mathrm{~min}$ in advance, to reach saturation. The three gas incubators were adjusted, and filled with $95 \% \mathrm{~N}_{2}+1 \%$ $\mathrm{O}_{2}+4 \% \mathrm{CO}_{2}$. The cell culture medium was discarded, washed twice with phosphate-buffered saline (PBS) and incubated for hypoxia cultivation. It was followed by removal of liquid inside and oxygen-free liquid was added. Hypoxic cells were removed after $12 \mathrm{~h}$, oxygen-free supernatants were collected and washed with the PBS, then added into complete DMEM/F12 medium. The cells were cultivated with reoxygenation in the three gas incubators adjusted to $95 \%$ air $+5 \%$ $\mathrm{CO}_{2}$, after $12 \mathrm{~h}$ cells and oxygen culture supernatant were collected.

Grouping. The animals were randomly divided into the control, model, low concentration $(5 \mu \mathrm{g} / \mathrm{ml})$, moderate concentration $(10 \mu \mathrm{g} / \mathrm{ml})$ and high concentrations $(20 \mu \mathrm{g} / \mathrm{ml})$ groups. After dissolving kidney pills in saline, the different concentration criterions were adjusted in terms of quality, experiment was co-cultured with $1 \mathrm{ml}$ medicine. The control group was normal renal tubular epithelial cells, and the model group was applied with $1 \mathrm{ml}$ saline. The apoptotic rate was measured with flow cytometry and Jag2/Notch2/hes1 mRNA as well as protein expression with quantitative PCR and western blot analysis detection, respectively, at 1 , 3 and 7 days. Five samples were selected at each time-point, and results are the average values.

Detection of cell apoptosis by flow cytometry. The NRK-52E cells were seeded into six pore plates in accordance with $5 \times 10^{5}$ cells/pore density. The pancreatic cells $(200 \mu \mathrm{l})$ were added and centrifuged for $5 \mathrm{~min}$ at $800 \mathrm{x} \mathrm{g}$. The supernatant was discarded; washed with pre-cold PBS and centrifuged for $5 \mathrm{~min}$ at $800 \mathrm{x} \mathrm{g}$, and was repeated twice, followed by removal of the supernatant. Subsequently, 500- $\mu 1$ binding buffer suspension cells were added, $5 \mu 1$ Annexin V-FITC was added and mixed well. The cells were then incubated for $15 \mathrm{~min}$ at $4^{\circ} \mathrm{C}$, and $5 \mu \mathrm{l} \mathrm{PI}$ was added to homogenize the content in the dark at room temperature for 5-15 $\mathrm{min}$, before detection within $1 \mathrm{~h}$.

Quantitative PCR detection. Conventional TRIzol extraction of total RNA, spectrophotometer purity and concentration, led to the production of cDNA. The primer sequences were: Jag2 forward, 5'-TGTGGTTGGAAGCCTTGTCTG-3' and reverse, 5'-TGTCCAAATGAGTGGTCCGT-3'; Notch2 forward, 5'-CACCAACTTACGCATTAAACAAGAC-3' and
Table I. Comparison of cell apoptosis rate (\%).

\begin{tabular}{lccr}
\hline Groups & \multicolumn{1}{c}{1 day } & \multicolumn{1}{c}{3 days } & \multicolumn{1}{c}{7 days } \\
\hline Control & $0.3 \pm 0.1$ & $0.2 \pm 0.1$ & $0.2 \pm 0.1$ \\
Model & $26.5 \pm 4.2$ & $24.2 \pm 3.5$ & $20.1 \pm 3.2$ \\
Low concentration & $21.4 \pm 4.0$ & $20.2 \pm 3.6$ & $15.5 \pm 3.2$ \\
Moderate concentration & $13.2 \pm 3.3$ & $7.5 \pm 2.0$ & $3.4 \pm 1.1$ \\
High concentraition & $13.3 \pm 3.4$ & $7.6 \pm 2.2$ & $3.5 \pm 1.3$ \\
F-value & 7.524 & 9.623 & 12.522 \\
P-value & $<0.001$ & $<0.001$ & $<0.001$ \\
\hline
\end{tabular}

reverse, 5'-TCCGATTCACTGACAACAGACAC-3'; hes1 forward, 5'-TGCTTTCCTCATCCCCAATG-3' and reverse, 5'-GAAGGCGACACTGCGTTAGG-3'; the internal control $\beta$-actin forward, 5'-CGTTGACATCCGTAAAGACCTC-3' and reverse, 5'-TAGGAGCCAGGGCAGTAATCT-3'. The reaction system consisted of cDNA $(5 \mu \mathrm{l})$, target gene downstream primer $(100 \mathrm{pmol} / \mathrm{l}, 2 \mu \mathrm{l}), 10 \mathrm{X}$ buffer $(2.5 \mu \mathrm{l}), 10 \mathrm{mmol}$ dBTPs $(3 \mu 1)$, Taq enzyme $(1 \mu \mathrm{l})$, ultra-pure water to total volume of $25 \mu 1$. The reaction parameters included denaturation at $95^{\circ} \mathrm{C}$ for $1 \mathrm{~min}, 95^{\circ} \mathrm{C}$ for $15 \mathrm{sec}, 58^{\circ} \mathrm{C}$ for $20 \mathrm{sec}, 72^{\circ} \mathrm{C}$ for $20 \mathrm{sec}$ for 40 cycles in total, and a final extension at $72^{\circ} \mathrm{C}$ for $5 \mathrm{~min}$. The final solubility curve results were expressed in the ratio of the target gene and reference gene, using the $2^{-\Delta \Delta C q}$ formula.

Western blot assay. Total protein was extracted and quantified with Bradford assay. Western blotting was performed as described elsewhere (7). The primary antibody was used for incubation overnight at $4^{\circ} \mathrm{C}$. The following day, the membrane was washed three times with TBST at room temperature and the secondary antibody diluted with TBST 3,000 times, was used for incubation at room temperature $30 \mathrm{~min}$. It was then washed three times with TBST at room temperature. Subsequently, the chemiluminescence reaction was used for developing the protein images. Rabbit polyclonal JAG2 antibody (dilution, 1:500; cat. no. ab109627), rabbit polyclonal Notch2 antibody (dilution, 1:500; cat. no. ab8926), rabbit polyclonal Hes1 antibody (dilution, 1:500; cat. no. ab71559) and secondary goat anti-rabbit (HRP) IgG antibody (dilution, 1/2000; cat. no. ab6721) were all purchased from Abcam (Cambridge, MA, USA).

Statistical analysis. SPSS 19.0 (Chicago, IL, USA) was used to analyze the data. Quantitative data are shown as mean \pm standard deviation, the comparison among groups was shown by using single-factor ANOVA analysis, while repeated measures analysis of variance was applied in the intra-group. Qualitative data were compared with the number of cases or percentage $(\%), \chi^{2}$ test was used among groups. $\mathrm{P}<0.05$ was considered to indicate a statistically significant difference.

\section{Results}

Comparison of the rate of apoptosis. In addition to the control group, the other groups took longer to grow. The apoptotic rate decreased and at each time-point of the moderate 

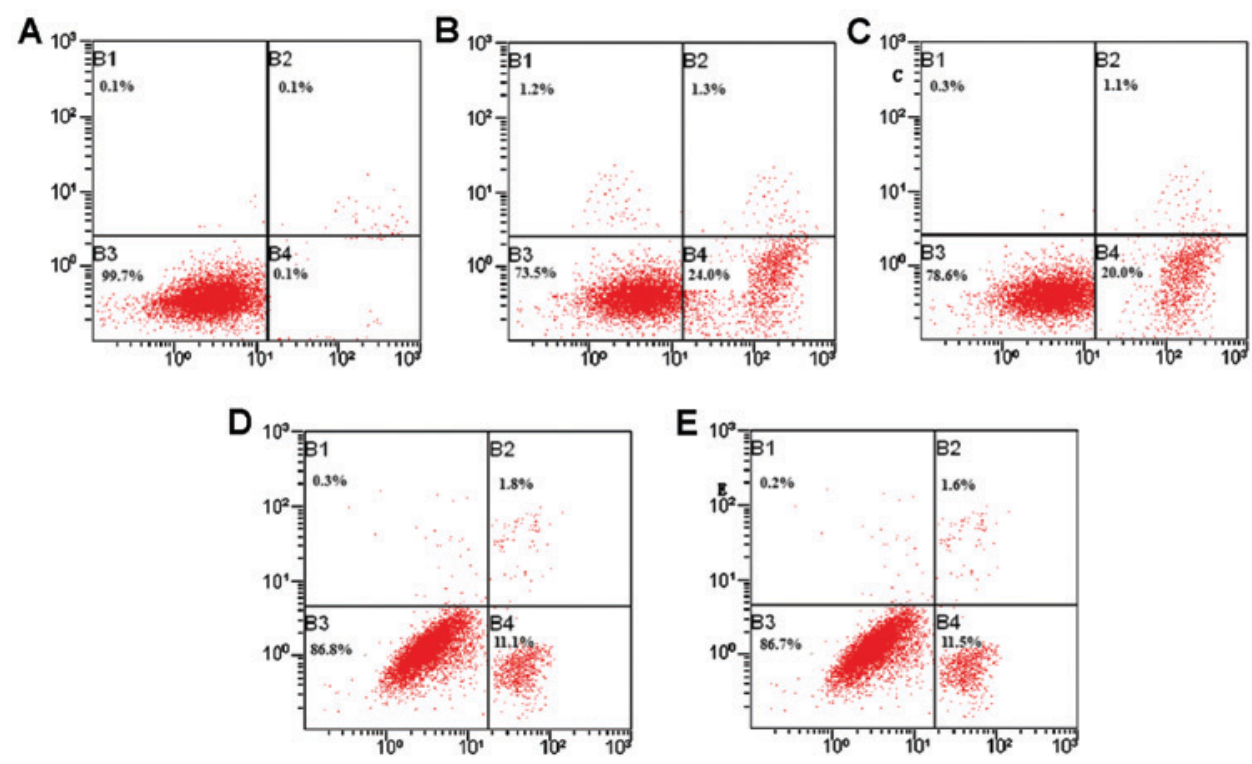

Figure 1. Cell apoptotic rate by flow cytometry. (A) Control, (B) model, (C) low concentration, (D) moderate concentration and (E) high concentration groups.

Table II. Comparison of mRNA Jag2/Notch2/hes1 expression levels.

\begin{tabular}{|c|c|c|c|c|c|c|c|c|c|}
\hline \multirow[b]{2}{*}{ Groups } & \multicolumn{3}{|c|}{ Jag2 } & \multicolumn{3}{|c|}{ Notch2 } & \multicolumn{3}{|c|}{ hes1 } \\
\hline & 1 day & 3 days & 7 days & 1 day & 3 days & 7 days & 1 day & 3 days & 7 days \\
\hline Control & $0.06 \pm 0.02$ & $0.05 \pm 0.01$ & $0.05 \pm 0.02$ & $0.04 \pm 0.01$ & $0.03 \pm 0.01$ & $0.03 \pm 0.01$ & $0.03 \pm 0.01$ & $0.02 \pm 0.01$ & $0.03 \pm 0.01$ \\
\hline Model & $0.42 \pm 0.06$ & $0.33 \pm 0.04$ & $0.28 \pm 0.04$ & $0.36 \pm 0.04$ & $0.30 \pm 0.03$ & $0.26 \pm 0.03$ & $0.34 \pm 0.03$ & $0.30 \pm 0.03$ & $0.25 \pm 0.03$ \\
\hline Low concentration & $0.40 \pm 0.05$ & $0.30 \pm 0.05$ & $0.25 \pm 0.03$ & $0.33 \pm 0.03$ & $0.27 \pm 0.04$ & $0.24 \pm 0.03$ & $0.31 \pm 0.03$ & $0.27 \pm 0.03$ & $0.22 \pm 0.03$ \\
\hline $\begin{array}{l}\text { Moderate } \\
\text { concentration }\end{array}$ & $0.32 \pm 0.04$ & $0.26 \pm 0.03$ & $0.19 \pm 0.03$ & $0.26 \pm 0.03$ & $0.20 \pm 0.02$ & $0.13 \pm 0.02$ & $0.25 \pm 0.03$ & $0.18 \pm 0.03$ & $0.12 \pm 0.03$ \\
\hline High concentration & $0.33 \pm 0.04$ & $0.28 \pm 0.04$ & $0.21 \pm 0.04$ & $0.27 \pm 0.03$ & $0.21 \pm 0.03$ & $0.15 \pm 0.02$ & $0.25 \pm 0.03$ & $0.20 \pm 0.03$ & $0.13 \pm 0.03$ \\
\hline F-value & 6.529 & 6.967 & 7.421 & 7.201 & 7.424 & 7.625 & 6.938 & 6.857 & 7.203 \\
\hline P-value & $<0.001$ & $<0.001$ & $<0.001$ & $<0.001$ & $<0.001$ & $<0.001$ & $<0.001$ & $<0.001$ & $<0.001$ \\
\hline
\end{tabular}

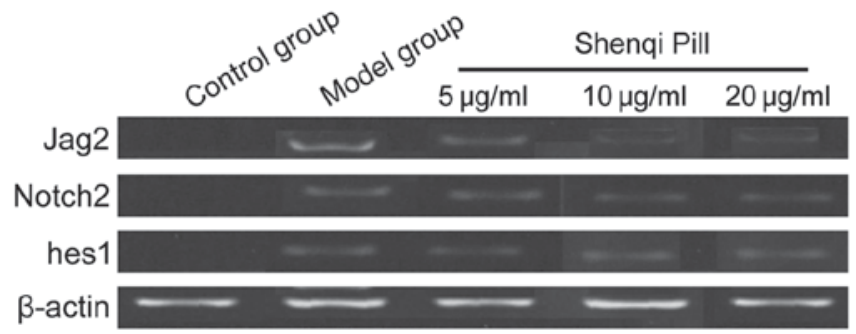

Figure 2. Detection of quantitative PCR method comparison of mRNA Jag2/Notch2/hes1 expression levels.

concentration and high concentration groups was significantly lower than that of the model and low concentration groups $(\mathrm{P}<0.05)$ (Table I and Fig. 1).

Comparison of mRNA Jag2/Notch2/hes1 expression levels. In addition to the control group, the other groups were grown with extension of time. The mRNA Jag2/Notch2/hes1 expression levels decreased and the expression levels of the mRNA $\mathrm{Jag} 2 /$ Notch2/hes1 of the concentration and high concentration group at each time-point was significantly lower than that of the model and low-dose groups, and the difference was significant $(\mathrm{P}<0.05)$ (Table II and Fig. 2).

Comparison of Jag2/Notch2/hes1 protein expression levels. In addition to the control group, the other groups took longer to grow. The Jag2/Notch2/hes1 protein expression levels decreased and the Jag2/Notch2/hes1 protein expression levels of concentration and high concentration group at each time-point was significantly lower than that of the model group and low-dose group $(\mathrm{P}<0.05)$ (Table III and Fig. 3).

\section{Discussion}

Notch signaling pathway is a major signaling pathway, which mediates the direct contact between the cells and also plays an important role in the regulation of cell growth, differentiation, tissue regeneration and the stability of intracellular environment. The Notch family consists of a group of highly conserved proteins including four cognate receptor Notch 1-4, 5 types of homolog ligand Delta-like 1,3 and 4, and Jagged 1 and 2, and two types of downstream target genes: The Hes and 
Table III. Comparison of Jag2/Notch2/hes1 protein expression levels.

\begin{tabular}{|c|c|c|c|c|c|c|c|c|c|}
\hline \multirow[t]{2}{*}{ Groups } & \multicolumn{3}{|c|}{ Jag2 } & \multicolumn{3}{|c|}{ Notch2 } & \multicolumn{3}{|c|}{ hes 1} \\
\hline & 1 day & 3 days & 7 days & 1 day & 3 days & 7 days & 1 day & 3 days & 7 days \\
\hline Control & $0.06 \pm 0.02$ & $0.05 \pm 0.01$ & $0.05 \pm 0.02$ & $0.04 \pm 0.01$ & $0.03 \pm 0.01$ & $0.03 \pm 0.01$ & $0.03 \pm 0.01$ & $0.02 \pm 0.01$ & $0.03 \pm 0.01$ \\
\hline Model & $0.48 \pm 0.07$ & $0.41 \pm 0.06$ & $0.36 \pm 0.06$ & $0.39 \pm 0.05$ & $0.32 \pm 0.04$ & $0.28 \pm 0.04$ & $0.36 \pm 0.04$ & $0.32 \pm 0.04$ & $0.27 \pm 0.03$ \\
\hline Low concentration & $0.44 \pm 0.05$ & $0.39 \pm 0.06$ & $0.33 \pm 0.05$ & $0.35 \pm 0.04$ & $0.30 \pm 0.04$ & $0.26 \pm 0.05$ & $0.33 \pm 0.04$ & $0.29 \pm 0.03$ & $0.24 \pm 0.04$ \\
\hline $\begin{array}{l}\text { Moderate } \\
\text { concentration }\end{array}$ & $0.35 \pm 0.04$ & $0.28 \pm 0.05$ & $0.20 \pm 0.04$ & $0.28 \pm 0.04$ & $0.22 \pm 0.03$ & $0.15 \pm 0.03$ & $0.26 \pm 0.04$ & $0.19 \pm 0.04$ & $0.15 \pm 0.03$ \\
\hline High concentration & $0.36 \pm 0.04$ & $0.29 \pm 0.06$ & $0.21 \pm 0.05$ & $0.29 \pm 0.04$ & $0.21 \pm 0.05$ & $0.16 \pm 0.03$ & $0.27 \pm 0.05$ & $0.20 \pm 0.03$ & $0.15 \pm 0.04$ \\
\hline F-value & 7.231 & 7.345 & 7.626 & 7.528 & 7.717 & 7.962 & 8.231 & 8.465 & 8.721 \\
\hline P-value & $<0.001$ & $<0.001$ & $<0.001$ & $<0.001$ & $<0.001$ & $<0.001$ & $<0.001$ & $<0.001$ & $<0.001$ \\
\hline
\end{tabular}

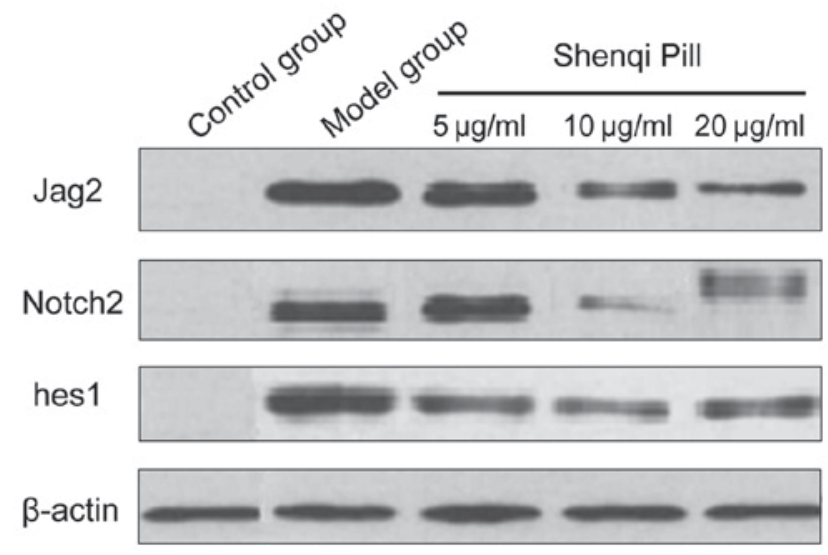

Figure 3. Jag2/Notch2/hes1 protein expression levels detected by western blot analysis.

Hey families. When tissue is subjected to hypoxia and drug damage, the activation of ligand expression would increase, which can result in the series of proteolytic cleavage of Notch receptors via the Notch receptor binding through cell to cell contact, and then release the Notch intracellular domain of activity; the Notch intracellular domain activity is transferred to the cell nucleus and combined with the transcription factor binding, starting the transcription of the target gene, regulating and controlling the cell proliferation $(7,8)$.

The Jinguishenqi pill is composed of $8 \mathrm{~g}$ Rehmannia, jujube peel, $4 \mathrm{~g}$ Chinese yam, $3 \mathrm{~g}$ each of Alisma, paeonol, Poria cocos and Cassia twig, and $1 \mathrm{~g}$ aconite. The original description: 'Rehmannia is sweet-warm, enriching yin and nourishing the kidney as the main drug, supplemented by acid tepid dogwood, nourishing liver and kidney, protecting vital essence with astringency; yam was sweet taste and neutral nature, good at strengthening spleen and tonifying kidney; plus a small amount of aconite and cinnamon which could warm kidney and enhance Yangqi, fill Mingmen real fire, conduct the fire back to its origin; accompanied with plantain regulating the waterways, draining water evil in renal; tuckahoe tonifying spleen, clearing damp and promoting diuresis; cortex mouton clearing liver fire', in various drug combinations $(9,10)$. The study indicated that in addition to the control group, each group grew for a longer time. The levels of apoptosis rate, Jag2/Notch2/hes1 mRNA and protein expression decreased and the levels of apoptosis, Jag2/Notch2/hes1 mRNA and protein expression of concentration and high concentration group were obviously lower than those of the model group and low dose group at each time-point, the difference was statistically significant. Glomerular apoptosis rate reached the peak when acute kidney injury occurred, and then decreased gradually. Jag2/Notch2/hes1 mRNA and protein expression levels were also decreased, these changes suggested that early intervention of acute kidney injury could reduce the extent of tissue damage, and be conducive to regeneration of tissue (11). Shenqi Pill could relieve the damage of renal tubular epithelial cells by inhibiting the activation and expression of Jag2/Notch2/hes1 signaling pathway. The different concentrations had different effects, low concentration slightly inhibited Jag2/Notch2/hes1 mRNA and protein expression, a suitable concentration such as $10-20 \mathrm{~g} / \mathrm{ml}$ could play a better supporting effect. The specific reasons for not getting improved effects by the increase of the concentration should be further analyzed.

The present study has confirmed the inhibitory effects of Shenqi Pill on Jag2/Notch2/hes1 signaling pathway by experiments in vitro. As the Shenqi pill is a compound drug, it was not possible to determine the specific components which played a major role. Application effects can be further explored in in vivo animal experiments or clinical studies.

\section{References}

1. Gao Y, Zeng Z, Li T, Xu S, Wang X, Chen Z and Lin C: Polydatin inhibits mitochondrial dysfunction in the renal tubular epithelial cells of a rat model of sepsis-induced acute kidney injury. Anesth Analg 121: 1251-1260, 2015.

2. Li K, Han Q, Yan X, Liao L and Zhao RC: Not a process of simple vicariousness, the differentiation of human adipose-derived mesenchymal stem cells to renal tubular epithelial cells plays an important role in acute kidney injury repairing. Stem Cells Dev 19: 1267-1275, 2010.

3. Cheng HT and Kopan R: The role of Notch signaling in specification of podocyte and proximal tubules within the developing mouse kidney. Kidney Int 68: 1951-1952, 2005.

4. Huang R, Zhou Q, Veeraragoo P, Yu H and Xiao Z: Notch2/Hes-1 pathway plays an important role in renal ischemia and reperfusion injury-associated inflammation and apoptosis and the $\gamma$-secretase inhibitor DAPT has a nephroprotective effect. Ren Fail 33: 207-216, 2011.

5. Nan Y, Zhou X, Liu Q, Zhang A, Guan Y, Lin S, Kong L, Han Y and Wang X: Serum metabolomics strategy for understanding pharmacological effects of ShenQi pill acting on kidney yang deficiency syndrome. J Chromatogr B Analyt Technol Biomed Life Sci 19: 102-103, 2015. 
6. Xiong X, Wang P, Li X and Zhang Y: Shenqi pill, a traditional Chinese herbal formula, for the treatment of hypertension: a systematic review. Complement Ther Med 23: 484-493, 2015.

7. Kramer J, Schwanbeck R, Pagel H, Cakiroglu F, Rohwedel J and Just U: Inhibition of Notch signaling ameliorates acute kidney failure and downregulates platelet-derived growth factor receptor $\beta$ in the mouse model. Cells Tissues Organs 201: $109-117,2016$.

8. Juillerat-Jeanneret L, Flohr A, Schneider M, Walter I, Wyss JC, Kumar R, Golshayan D and Aebi JD: Targeted $\gamma$-secretase inhibition to control the Notch pathway in renal diseases. J Med Chem 58: 8097-8109, 2015
9. Long YL and Li ZM: Effect of jingui shenqi pill and its disassembled recipes on ovarian functions in shen yang deficiency female rats. Zhongguo Zhong Xi Yi Jie He Za Zhi 33: 967-971, 2013 (In Chinese).

10. Xu CP, Zhu QJ, Song J, Li Z and Zhang D: Acceleration of Jingui Shenqi Pill on the testis telomerase activity in mice of Shen-yang deficiency. Zhongguo Zhong Xi Yi Jie He Za Zhi 33: 252-255, 2013 (In Chinese)

11. Sörensen-Zender I, Rong S, Susnik N, Zender S, Pennekamp P, Melk A, Haller H and Schmitt R: Renal tubular Notch signaling triggers a prosenescent state after acute kidney injury. Am J Physiol Renal Physiol 306: F907-F915, 2014. 